\title{
Early outcomes of cardiac surgical patients who developed COVID-19 in the peri-operative period-results from an online survey
}

\author{
George Jose Valooran ${ }^{1} \cdot$ Kirun Gopal $^{2} \cdot$ Rajesh Jose $^{2} \cdot$ Praveen Kerala Varma $^{2}$
}

Received: 6 July 2021 / Revised: 15 July 2021 / Accepted: 16 July 2021 / Published online: 17 September 2021

(c) Indian Association of Cardiovascular-Thoracic Surgeons 2021

\section{Introduction}

Coronavirus infectious disease 2019 (COVID-19) pandemic has resulted in global disruption of cardiac surgical practice. More worrisome is the impact of COVID on cardiac surgical patients, especially in the immediate post-operative period. Although several smaller studies $(n<35)$ have reported higher surgical mortality in the setting of peri-operative COVID-19 infection, outcomes specific to cardiac surgery are sparse [1]. COVID Surg Collaborative analyzed the outcome of 1128 surgical patients with peri-operative COVID-19 positivity across various specialties in 235 hospitals spread over 25 countries. They reported an overall 30 -day mortality of $23.8 \%$ [2]. The recent United Kingdom (UK)-based study analyzed 755 adult patients undergoing cardiac surgery, of which $53(7.0 \%)$ patients had peri-operative COVID diagnosis. They reported a 30-day mortality rate of $24.5 \%$ vs. $3.5 \%(p=<0.0001)$ in COVID-positive versus COVID-negative cases [1], which sheds some light on the impact of the pandemic on cardiac surgical outcomes. There is a paucity of data from India regarding its impact on outcomes after cardiac surgery. Hence, we undertook an online questionnaire to study the early outcomes of patients who underwent cardiac surgery and who developed COVID during the in-hospital admission period.

Praveen Kerala Varma

varmapk@gmail.com

1 Department of Cardiothoracic Surgery, Government General Hospital, Ernakulam, India

2 Department of Cardiothoracic Surgery, Amrita Institute of Medical Sciences, Amrita Viswa Vidyapeetham (Amrita University), Kochi, India

\section{Methods}

The anonymous survey questionnaire comprised of thirteen queries designed to collect outcome data of cardiac surgical procedures (both adult and pediatric). The responders were encouraged to furnish the data available from the outset of pandemic (World Health Organization (WHO) declaration of pandemic, January 2020 [1]) till date.

The weblink (http://play.google.com/store/apps/details? $\mathrm{id}=$ com. surveyheart) to the questionnaire was forwarded over emails (email IDs retrieved from corresponding author's personal database) to the practicing fellow cardiac surgeons. The link was also forwarded as what's-app messages in the informal loco-regional and national groups of cardiac surgeons to augment the reach. The link remained active from 23/05/2021 to 23/06/2021. A single reporting person per center was advised to complete the questionnaire in order to avoid duplication of data.

\section{Results}

Of the 67 responses derived, 4 were deemed incomplete or vague and were excluded. The remaining 63 responses were analyzed. Pooling the responses, 330 patients were reported to have documented peri-operative COVID as indicated by a positive antigen/real-time reverse transcription-polymerase chain reaction (RTPCR)/TrueNat ${ }^{\mathrm{TM}}$ Beta $\mathrm{CoV}$ and severe acute respiratory syndrome coronavirus 2 (SARS-CoV-2) point-of-care test during the same surgical admission and underwent cardiac surgery. Sixty-six patients out of 330 operated cases had in-hospital resuscitations, re-explorations, or major adverse events (re-intubations/mortality/stroke/ repeat revascularizations) before discharge (20\%). Of the 330 positive patients who underwent cardiac surgery, 47 patients (14.2\%) had in-hospital mortality. A total of 264 patients had uneventful recovery $(264 / 330,80 \%)$. 


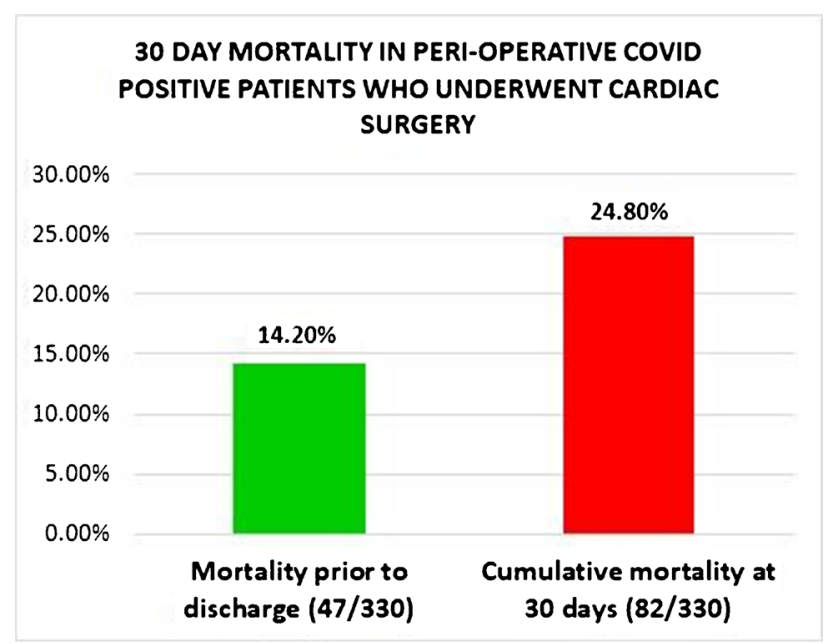

Fig. 1 Thirty-day mortality in peri-operative COVID-positive patients who underwent cardiac surgery

There were 47 re-admissions requiring intervention or mechanical ventilation within the first 30 days following discharge. Another 35 patients expired in the first month after discharge, totaling 82 post-operative deaths with a cumulative 30 -day mortality of $24.8 \%$ (Fig. 1). The cause of mortality was perceived to be respiratory (33.33\%) followed by multi-organ dysfunction syndrome (MODS) $(15.87 \%)$, while cardiac- and cardiac surgery-related causes were reported to be $5.97 \%$. The average hospital stay in COVID-positive patients after cardiac surgery was reported to be $10-21$ days by $28.57 \%,<10$ and $>21$ days respectively by $28.57 \%$ and $17.46 \%$ responders. There was no extracorporeal membrane oxygenation (ECMO) initiation reported and $84.13 \%$ reported that they could not comment reliably on the association of cardio-pulmonary bypass (CPB) and poorer surgical outcomes. Figure $2 \mathrm{~A}$ depicts the reported case numbers during the

\section{A- AVERAGE CASE NUMBERS PER MONTH}

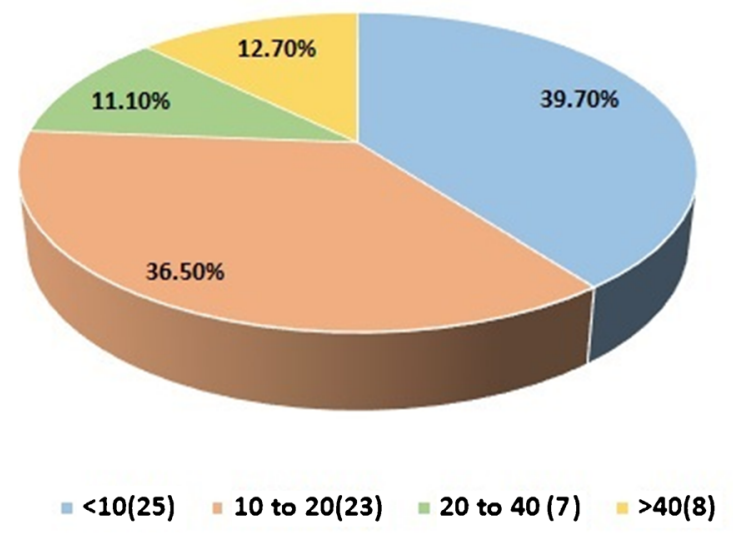

pandemic. Majority of the procedures (37.1\%) performed during the pandemic were reported to be of urgent nature followed by early $(27.42 \%)$, elective $(19.35 \%)$, and salvage $(15.32 \%)$ (Fig. 2B). Only $1.16 \%(2 / 63)$ of the surgeons, based on their judgments, suggested suspending all cardiac surgical procedures during the pandemic while only $13 / 63(7.51 \%)$ surgeons recommended the conduct of elective cases.

\section{Discussion}

The Indian Association of Cardio-Thoracic Surgeons (IACTS) had provided the framework for pre-operative workup, case selection protocols, and COVID-positive triage systems to support cardiac surgical practice in India [3]. With the second wave hitting India and the anticipation of the third wave around the corner, it would be imperative to perform outcome analyses of the surgical results to synthesize new and modify the existing practice protocols. Although anonymous surveys provide non-verifiable data, they have the advantages of faster data generation and enhanced transparency of reporting to reflect the current practice trends.

The cumulative 30-day mortality (24.8\%) as documented by the present survey parallels the mortality data furnished by the UK study pertaining to cardiac surgery $(24.5 \%)$ and that of the COVID Surg Collaborative reporting various surgical specialties $(23.8 \%)$ [2,3]. This further support the observations of our surgeons that the cause of death in COVID-positive cardiac surgical patients is primarily not cardiac or cardiac surgery related. The need for multiple and rigorous pre-operative screening for COVID thus assumes paramount importance and might be decisive in determining surgical outcomes.

\section{B-TYPES OF CARDIAC SURGERY PERFORMED DURING} PANDEMIC

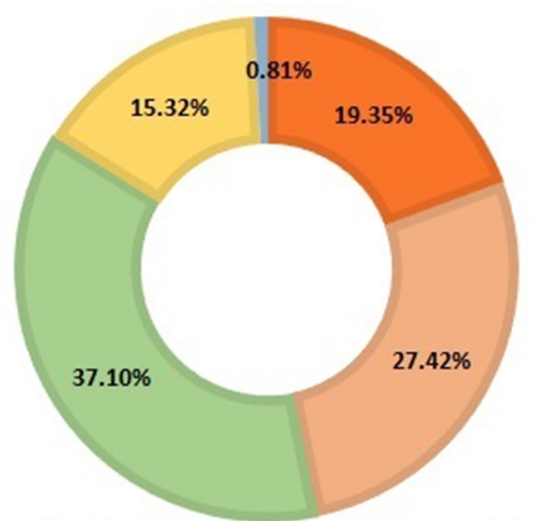

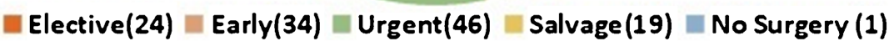

Fig. 2 A Average case numbers in the centers per month during the pandemic. B Types of cases performed in the centers during the pandemic 


\section{Study limitation}

The limitations of the study include the non-verifiable nature of the survey data, wide variability in the nature of cardiac surgical procedures reported, and the lack of input on surgical risk and limited representation of national data. More robust data collection and reporting are needed to verify this data.

\section{Conclusions}

The development of peri-operative COVID in cardiac surgery was associated with very high mortality. We believe that it is time for us as a fraternity to introspect and adapt to the changing practice environment, furthered by research and revision of guidelines. "In the rush to get back to normal, use this time to decide which parts of normal are worth rushing back to" (Dave Hollis, French author).

Acknowledgements We thank all the cardiothoracic surgeons who participated in the survey.

\section{Declarations}

Ethics approval Not obtained as this was an anonymous survey.
Informed consent Not required.

Animal and human rights Not required being that this study was based on an anonymous survey.

Conflict of interest The authors declare no competing interests.

\section{References}

1. Sanders J, Akowuah E, Cooper J, et al. Cardiac surgery outcome during the COVID-19 pandemic: a retrospective review of the early experience in nine UK centres. J Cardiothorac Surg. 2021;16:43.

2. COVIDSurg Collaborative. Mortality and pulmonary complications in patients undergoing surgery with perioperative SARS-CoV-2 infection: an international cohort study. Lancet. 2020;396:27-38.

3. Hiremath CS, Yadava OP, Meharwal ZS, Iyer KS, Velayudhan B. IACTS guidelines: practice of cardiovascular and thoracic surgery in the COVID-19 era. Indian J Thorac Cardiovasc Surg. 2020;36:438-50.

Publisher's note Springer Nature remains neutral with regard to jurisdictional claims in published maps and institutional affiliations. 